\title{
The Sol-Gel Transition and the Liquid-Liquid Phase Separation in Poly(vinyl chloride) Solutions ${ }^{\dagger}$
}

\author{
Kazuo KAwANishi, Yuji TAKeDA, and Takashi INOUE* \\ Department of Textile and Polymeric Materials, \\ Tokyo Institute of Technology, \\ Ookayama, Meguro-Ku, Tokyo 152, Japan
}

(Received December 17, 1985)

\begin{abstract}
The dynamics of the sol-gel transition in poly(vinyl chloride) $/ \gamma$-butyrolactone system was investigated by light scattering. The dynamical studies confirmed that this system has an UCST (upper critical solution temperature). In the phase diagram, temperature $v s$. concentration, the spinodal curve crosses the sol-gel transition curve. This situation has been predicted by Coniglio et al. as a result of the site-bond correlated-percolation problem. The gelation takes place both above and under the spinodal curve, suggesting two modes of gelation: one is accompanied by a spinodal decomposition and the other takes place without liquid-liquid phase separation. In the former case, the dynamics of phase separation at an early stage of gelation is well described within the framework of Cahn's linearized spinodal decomposition theory. It is noted that, in order to understand the entire mechanism of sol-gel transition, one should clarify the three dynamical aspects on the liquid-liquid phase separation, percolation-type association, and liquid-solid transition.
\end{abstract}

KEY WORDS Poly(vinyl chloride)/ $\gamma$-Butyrolactone / Sol-Gel Transition / Light Scattering / Liquid-Liquid Phase Separation / Percolation / LiquidSolid Transition /

It is well known that polymer solutions, such as poly(vinyl alcohol) (PVA) in water, polyacrylonitrile (PAN) in a mixed solvent of $\mathrm{N}, \mathrm{N}$-dimethyl formamide/ethylene glycol, and poly(vinyl chloride) (PVC) in $\gamma$-butyrolactone, lose their fluidity upon cooling, i.e., the solution changes from a sol to a gel. It is generally agreed that gelation is the result of network formation. However the nature of the tie points in the network is the subject of some controversy.

A widely accepted view considers them to be polymer crystallites. This concept has been supported by extensive discussions on the "gel melting" behavior in terms of the thermody- namics of melting of crystallites. ${ }^{1-3}$ On the other hand, $\mathrm{Paul}^{4}$ and Labudzinska et al. ${ }^{5}$ suggested that the gelation originates from phase separation into polymer-rich and polymer-poor regions. However they could not propose a clear structural model for network formation. A few years later, Prins et al. , $^{6,7}$ applied the concept of spinodal decomposition to the gelation process and interpreted the origin of network structure in terms of the connectivity of the polymer-rich phase. They successfully applied their concept to aqueous agarose solutions. On the same line as theirs, we have undertaken a more quantitative study on the aqueous PVA solutions, resulting in a

${ }^{\dagger}$ Presented in part at the 34th Polymer Symposium, Society of Polymer Science, Japan, September 1985 [Polym. Prepr. Jpn., 34, 2141 (1985)].

* To whom correspondence should be addressed. 
somewhat different conclusion. ${ }^{8}$

An important conclusion from our results is that there certainly exists a mode of gelation in which spinodal decomposition takes place at the early stage of gelation; however the spinodal decomposition is not a necessary condition but also a sufficient one for the sol-gel transition. So, in order to understand the origin of sol-gel transition, we have to go back once again to the classic idea of the gelation by hydrogen-bonding type association by Eldridge and Ferry ${ }^{9}$ and also back to the concept of gelation induced by crystallization mentioned above.

For the gelation of PVC solution, a number of crystallographic studies has been accumulated by Keller and co-workers. ${ }^{10-12}$ This is the reason why we paid attention to the PVC/ $\gamma$-butyrolactone system and undertook dynamical studies on the sol-gel transition.

We report the results of light scattering studies on the dynamic behavior of $\mathrm{PVC} / \gamma$ butyrolactone system undergoing a sol-gel transition. We describe the phase diagram in terms of liquid-liquid phase equilibrium and sol-gel transition. From these results, we discuss the mechanism of the sol-gel transition.

\section{EXPERIMENTAL}

PVC, a commercial polymer, was supplied by the Mitsui Toatsu Chemicals Co., Ltd. (Vinichlon HS-H; degree of polymerization 3200 , polymerized at $37^{\circ} \mathrm{C}$ ). PVC $/ \gamma$-butyrolactone solutions were made up in a NMR tube with inner diameter of $6.7 \mathrm{~mm}$. The polymer was dissolved by gradual heating to 90 $100^{\circ} \mathrm{C}$, taking about $2 \mathrm{~h}$.

The solution was quenched in a water-bath kept at a constant temperature. After standing for $12 \mathrm{~h}$, the test tube was turned upside down. If the level meniscus deformed under its own weight, we judged that the solution was still a sol. When the meniscus could not be seen to deform, we judged that the system had gelled. Thus visual investigations on sol-gel transi- tions were carried out for solutions of various polymer concentrations at temperatures ranging from $0^{\circ} \mathrm{C}$ to $70^{\circ} \mathrm{C}$.

Similar temperature-drop experiments were carried out in a light scattering photometer, a Shimazu Light Scattering Photometer, Model PG-21, whose light source was a He-Ne 632.8 $\mathrm{nm}$ laser. When the sample tube was put into a thermoregulated silicone oil bath in the light scattering apparatus, the solution underwent a rapid temperature drop to the desired temperature. During the isothermal phase separation process, the change in angular distribution of scattered light intensity with time was measured.

\section{RESULTS AND DISCUSSION}

A sol-gel transition curve is shown in Figure 1. An open circle in Figure 1 means that the solution gelled after standing $12 \mathrm{~h}$. A cross means that the solution was still a sol. On standing longer than $12 \mathrm{~h}$, the sol-gel transition curve was almost identical with that in Figure 1.

Figure 2 is a typical example of the change of scattered intensity with time at various values of $q$, where the magnitude of the scattering vector $q$ is given by $q=(4 \pi / \lambda) \sin (\theta / 2), \lambda$ being the wavelength of the light in the solutions and $\theta$ the scattering angle. In the initial

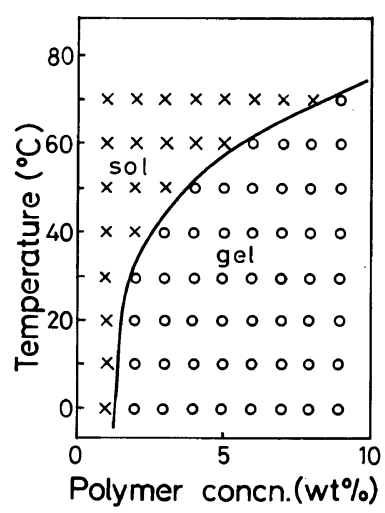

Figure 1. Sol-gel transition curve of PVC/ $\gamma$-butyrolactone system. 


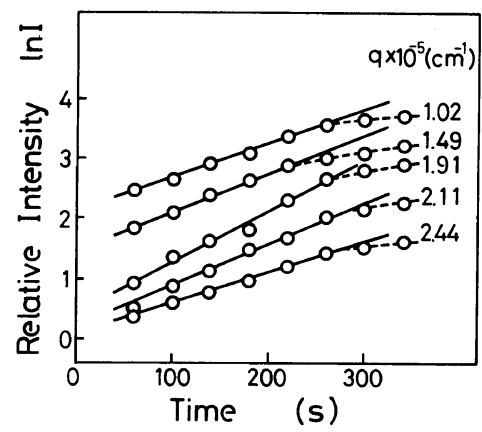

Figure 2. Time dependence of scattered light intensity at various values of $q$ from a $4 \mathrm{wt} \%$ solution quenched to an isothermal setting at $25^{\circ} \mathrm{C}$, demonstrating the exponential character of $I(t)$ at the initial stage.

stage of phase separation, the scattered intensity increases exponentially with time. In the later stages, the intensity deviates increasingly from the exponential curve.

According to Cahn's linear theory of spinodal decomposition, the exponential increase of the scattered intensity is described by

$$
I(q, t) \propto \exp [2 R(q) \cdot t]
$$

where $I$ is the scattered intensity, $t$ is the time after the initiation of the spinodal decomposition, and $R(q)$ is the growth rate of concentration fluctuation having the wavenumber $q$. $R(q)$ is given by

$$
R(q)=-M \cdot q^{2}\left(\frac{\partial^{2} f}{\partial c^{2}}+2 K q^{2}\right)
$$

where $M$ is the mobility, $K$ is the concentration-gradient energy coefficient, $f$ is the free energy of mixing, and $c$ is the concentration of the solution. ${ }^{13}$ According to eq 1 , a plot of $\ln I$ $v s$. $t$ at fixed $q$ should yield a straight line of slope $2 R(q)$. A linear relationship is realized for the initial stage of phase separation, as shown in Figure 2. This indicates that the initial stage can be described by the linearized spinodal decomposition theory. Linear results were also obtained for solutions of other concentrations at various temperatures.

Figure 3 shows plots of $R(q) / q^{2} v s . q^{2}$. As expected from eq 2 , the plots are good straight

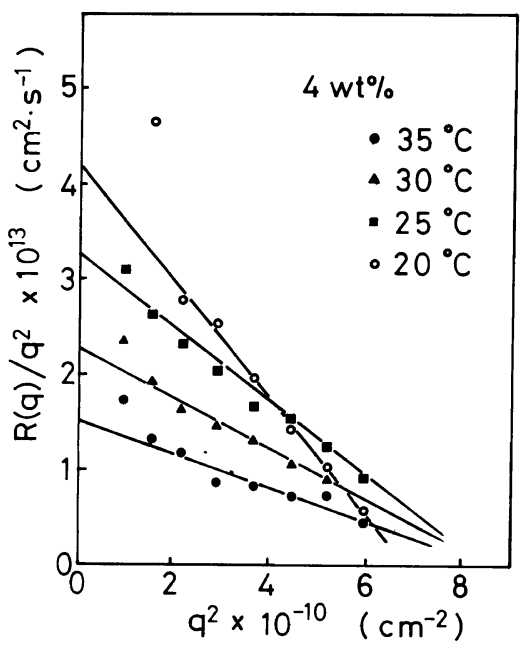

Figure 3. Plots of $R(q) / q^{2} v s . q^{2}$ for $4 \mathrm{wt} \%$ solutions quenched to the various indicated temperatures.

lines, indicating again that the initial stage can be described within the framework of the linear theory. From the plots, one can obtain such characteristic parameters as $q_{\mathrm{m}}, q_{\mathrm{c}}$, and $\tilde{D}$, describing the dynamics of phase separation. $q_{\mathrm{c}}$ is the critical (maximum) wavenumber of fluctuations which can grow. $q_{\mathrm{m}}$ is the most probable wave number of fluctuations having the highest rate of growth. According to eq 2, $q_{\mathrm{c}}$ is given by the intercept on the $q^{2}$ axis. $q_{\mathrm{m}}$ is calculated from the relation $q_{\mathrm{m}}^{2}=(1 / 2) q_{\mathrm{c}}^{2}$. The apparent diffusion coefficient $\tilde{D}$ defined by $\tilde{D}=-M \cdot\left(\partial^{2} f / \partial c^{2}\right)$ is given by the intercept on the vertical axis. The parameters thus estimated are summarized in Table I.

Figure 4 shows the temperature dependence of $\tilde{D}$ for various polymer concentrations. From the intercept on the temperature axis, one can estimate the spinodal temperature $T_{\mathrm{s}}$ at which $\tilde{D}$ is zero. A plots of $T_{\mathrm{s}} v s$. concentration, the spinodal curve, is given in Figure 5. The sol-gel transition curve of Figure 1 is reproduced in the phase diagram of Figure 5. From these scattering studies, it is unambiguous that the phase diagram of the $\mathrm{PVC} / \gamma$ butyrolactone system is of the UCST (upper critical solution temperature) type. 
Table I. Characteristic parameters describing spinodal decomposition of poly(vinyl chloride) $/ \gamma$-butyrolactone system

\begin{tabular}{|c|c|c|c|c|c|c|}
\hline $\begin{array}{c}\text { Polymer } \\
\text { concentration }\end{array}$ & Temp & $q_{\mathrm{m}} \times 10^{-6}$ & $q_{\mathrm{c}} \times 10^{-5}$ & $R\left(q_{\mathrm{m}}\right) \times 10^{3}$ & $-\tilde{D} \times 10^{13}$ & $T_{\mathrm{s}}$ \\
\hline $\mathrm{wt} \%$ & ${ }^{\circ} \mathrm{C}$ & $\mathrm{cm}^{-1}$ & $\mathrm{~cm}^{-1}$ & $\mathrm{~s}^{-1}$ & $\mathrm{~cm}^{2} \mathrm{~s}^{-1}$ & ${ }^{\circ} \mathrm{C}$ \\
\hline \multirow{3}{*}{2} & 20 & 1.26 & 1.78 & 6.00 & 3.60 & \multirow{3}{*}{37.} \\
\hline & 25 & 1.50 & 2.12 & 3.75 & 2.28 & \\
\hline & 30 & 1.83 & 2.59 & 2.83 & 1.96 & \\
\hline \multirow{4}{*}{4} & 20 & 1.36 & 1.92 & 8.10 & 4.18 & \multirow{4}{*}{43.8} \\
\hline & 25 & 1.45 & 2.05 & 7.85 & 3.25 & \\
\hline & 30 & 1.49 & 2.10 & 7.55 & 2.26 & \\
\hline & 35 & 1.91 & 2.70 & 3.59 & 1.51 & \\
\hline \multirow{3}{*}{6} & 25 & 1.30 & 1.84 & 4.90 & 3.50 & \multirow{3}{*}{38.1} \\
\hline & 30 & 1.93 & 2.73 & 4.00 & 1.94 & \\
\hline & 35 & 2.00 & 2.83 & 1.90 & 1.05 & \\
\hline
\end{tabular}

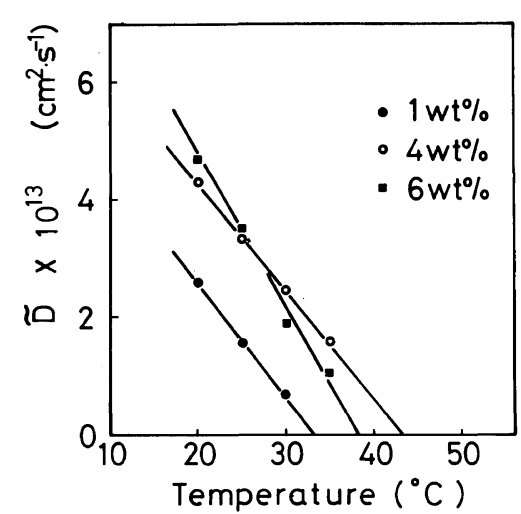

Figure 4. Temperature dependence of the apparent diffusion coefficient $\tilde{D}$.

In Figure 5, the spinodal curve and sol-gel transition curve divide the phase diagram into four regions as labeled from (i) to (iv). In region (i), the solution is a homogeneous sol. In region (ii), phase separation by spinodal decomposition takes place but the solution does not change to a gel. In region (iii), both gelation and spinodal decomposition occur. In other words, the gelation is accompanied by liquid-liquid phase separation through spinodal decomposition. In region (iv), gelation takes place without liquid-liquid phase separation. The presence of region (ii) implies that

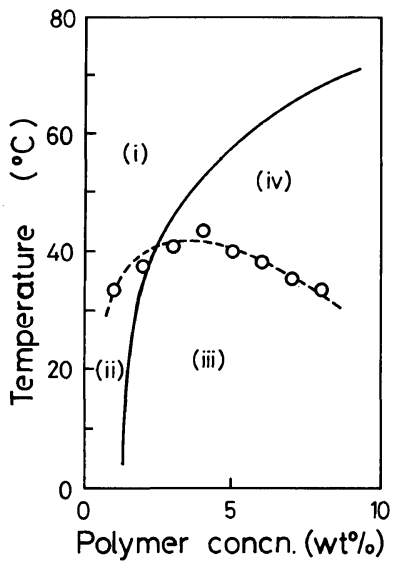

Figure 5. Sol-gel transition curve (solid line) and spinodal (broken line).

the liquid-liquid phase separation by the spinodal decomposition does not always induce gelation. In region (iii), it is expected that the supermolecular structure in the gel would be much influenced by liquid-liquid phase separation in the early stage of gelation. The presence of region (iv) means that spinodal decomposition is not a necessary condition for gelation.

It should be noted that no coarsening of the supermolecular structure was observed in the late stages of phase separation in region (iii), even after $12 \mathrm{~h}$. These results suggest that 


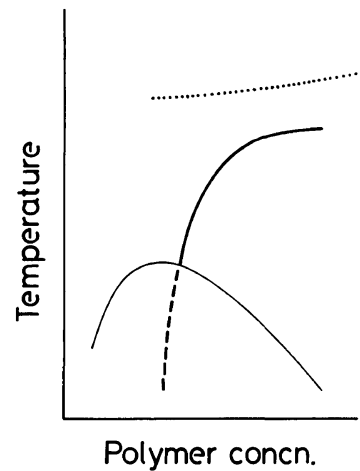

Figure 6. The coexistence curve (thin solid line); the sol-gel transition curve (thick solid line) and that continued into the two-phase region (broken line), predicted theoretically ${ }^{14}$; and the liquid-solid transition curve (dotted line), i.e., the melting point depression curve. ${ }^{16}$

further growth of concentration fluctuations may be suppressed in the late stages of phase separation. This is probably due to a hydrogen-bonding type association and/or the crystallite formation in the polymer-rich phase, resulting in the immobilization of polymer chains. ${ }^{8}$ We believe that this type of behavior must be characteristic of the spinodal decomposition in solutions of gel-forming polymers.

The last but very important point to be discussed is the presence of the gel region (iii) itself. A modern theory of gelation developed by Coniglio et al. ${ }^{14}$ as the site-bond correlatedpercolation problem predicts that the sol-gel transition curve will cross the coexistence (liquid-liquid phase equilibrium) curve, as is reproduced in Figure 6. From a theoretical point of view, gelation does not takes place in the two-phase region because of macroscopic phase separation into two coexisting phases. Hence the theoretical sol-gel transition curve is continued into the two-phase region by the broken line in Figure 6. On the contrary, in our PVC/ $\gamma$-butyrolactone system, as well as in aqueous PVA solution, ${ }^{8}$ gelation occurs even in the two-phase region; the gelation in region (iii).

As has been already mentioned, spinodal decomposition results in the development of the interconnected structure of polymer-rich and polymer-poor regions. In our PVC system, it is a fairly fast rate process (rate constant $R\left(q_{\mathrm{m}}\right)$ is order of $10^{-3} \mathrm{~s}^{-1}$; see Table I) and gelation follows. Therefore, when gelation takes place in a polymer-rich region, the whole system should be gelled unless the connectivity of the polymer-rich region is interrupted. This is the reason why the experimental sol-gel transition curve continues into the two-phase region.

We have found qualitative agreement between our experimental results and the percolation theory. However, a fundamental problem is the "site-bond". It may be a kind of hydrogen-bonding type association of polymer chains but this should be verified experimentally.

On the other hand, Keller and co-workers $^{10-12}$ have accumulated crystallographic evidence for the formation of crystallite in PVC gel. Gelation by crystallite formation is probably a phenomenon which takes place at a very late stage of sol-gel transition. ${ }^{8}$ The crystallization (liquid-solid transition) should be taken into account. Hence, in Figure 6, we have added a liquid-solid transition curve following the Flory's formulation of the melting point depression, ${ }^{15}$ schematically by dotted line. $^{16}$

In summary, we note that, in order to understand correctly the nature of sol-gel transition, attention should be directed to three fundamental phenomena:

(a) liquid-liquid phase separation,

(b) percolation-type association, and

(c) liquid-solid transition.

Especially, we have to clarify the relative rates of respective phenomena, in other words, the competitive situation of these three phenomena.

\section{CONCLUSIONS}

We investigated the sol-gel transition in $\mathrm{PVC} / \gamma$-butyrolactone system by light scatter- 
ing. We confirmed that the phase diagram of the PVC system is of the UCST type. The solgel transition curve and the spinodal curve obtained by dynamical studies divide the phase diagram into four regions:

(i) the homogeneous sol region,

(ii) the sol region under the spinodal curve,

(iii) the gel region under the spinodal curve, and

(iv) the gel region above the spinodal curve.

The presence of regions (ii) and (iv) implies that the spinodal decomposition is not a necessary condition but also a sufficient one for the sol-gel transition. Nevertheless, there exists a mode of gelation in which spinodal decomposition takes place at an early stage in gelation; hence the supermolecular structure of the gel may be governed by the liquid-liquid phase separation. That is, we have obtained a conclusion almost identical to that in aqueous PVA solutions. ${ }^{8}$ We believe that this type of behavior should be general in solutions of gelforming polymers.

Finally we note that, in order to understand the entire mechanism of the sol-gel transition, one should clarify the three dynamical aspects on the liquid-liquid phase separation, the percolation-type association, and the liquid-solid transition.

\section{REFERENCES AND NOTE}

1. A. Takahashi, N. Nakamura, and I. Kagawa, Polym. J., 3, 207 (1972).

2. S. Matsuzawa, K. Yamaura, R. Maeda, and K. Ogasawara, Makromol. Chem., 180, 229 (1979).

3. K. Ogasawara, T. Nakajima, K. Yamaura, and S. Matsuzawa, Colloid Polym. Sci., 254, 982 (1976).

4. D. R. Paul, J. Appl. Polym. Sci., 11, 439 (1976).

5. A. Labudzinska and A. Ziabicki, Kolloid Z.-Z. Polym., 243, 21 (1971).

6. E. Prins and W. Prins, Macromolecules, 6, 888 (1973).

7. G. T. Feke and W. Prins, Macromolecules, 7, 527 (1974).

8. M. Komatsu, T. Inoue, and K. Miyasaka, J. Polym. Sci., Polym. Phys. Ed., 24, 303 (1986).

9. J. E. Eldridge and J. D. Ferry, J. Phys. Chem., 58, 992 (1954).

10. S. J. Guerrero, A. Keller, P. L. Soni, and P. H. Geil, J. Macromol. Sci. Phys., B20, 161 (1981).

11. S. J. Guerrero and A. Keller, J. Macromol. Sci. Phys., B20, 167 (1981).

12. S. J. Guerrero, D. Meader, and A. Keller, $J$. Macromol. Sci. Phys., B20, 185 (1981).

13. J. W. Cahn, J. Chem. Phys., 42, 93 (1965).

14. A. Coniglio, H. E. Stanley, and W. Klein, Phys. Rev. Lett., 42, 518 (1979).

15. P. J. Flory, "Principles of Polymer Chemistry," Cornel Univ. Press, Ithaca, N. Y., 1953, Chapter 13.

16. The liquid-solid transition curve is located above the percolation curve. This is based upon a quantitative calculation. It's detail will be described in a forthcoming paper. 